Nervenarzt 2019 $\cdot 90: 1125-1134$ https://doi.org/10.1007/s00115-019-00808-9 Online publiziert: 28. Oktober 2019 (C) Der/die Autor(en) 2019

\author{
E.-L. Brakemeier ${ }^{1,2,3} \cdot$ S. C. Herpertz ${ }^{4}$ \\ ${ }^{1}$ Lehrstuhl Klinische Psychologie und Psychotherapie, Institut für Psychologie, Universität Greifswald, \\ Greifswald, Deutschland \\ ${ }^{2}$ Klinische Psychologie und Psychotherapie, Fachbereich Psychologie, Philipps-Universität Marburg \\ Marburg, Deutschland \\ ${ }^{3}$ Schön Klinik Bad Arolsen, Bad Arolsen, Deutschland \\ ${ }^{4}$ Klinik für Allgemeine Psychiatrie, Universitätsklinikum Heidelberg, Heidelberg, Deutschland
}

\title{
Innovative Psychotherapie- forschung: auf dem Weg zu einer evidenz- und prozessbasierten individualisierten und modularen Psychotherapie
}

tivität ist mit der somatischer Behandlungsverfahren vergleichbar und entsprechend hat sie als evidenzbasierte Behandlungsempfehlung der 1 . Wahl bei fast allen psychischen Erkrankungen Einzug in nationale und internationale Leitlinien gehalten. Es steht uns eine Vielfalt an empirisch untersuchten und professionell durchgeführten Psychotherapien zur Verfügung, die sich hinsichtlich ihrer Wirksamkeit im Durchschnitt nicht wesentlich zu unterscheiden scheinen. Dies scheint insbesondere für die Psychotherapie der Depressionen zuzutreffen (z. B. [3]) und wird im Diskurs der aktuellen Psychotherapiedebatte auch als Bestätigung des „Äquivalenzparadoxons“ bezeichnet [60]. Die kognitive Verhaltenstherapie (KVT) ist jedoch ohne Zweifel die am besten untersuchte Psychotherapie, weshalb die Evidenzbasierung hier als am robustesten bezeichnet werden kann [3].

\section{) Innovative Psychotherapie- forschung zur Überwindung der Kluft zwischen Forschung und Praxis}

Soweit die positive Zusammenfassung zur Wirksamkeit der Psychotherapie, welche allerdings durch eine Reihe von Limitationen eingeschränkt wird. Wir beschränken uns auf die unserer Meinung nach wichtigsten sechs Probleme:

1. Hoher Prozentsatz an Nonresponse, Nonremission und Rückfällen Trotz der durchschnittlichen Wirksamkeit zeigen Studien zu den etablierten Psychotherapien durchgängig Prozentanteile von mindestens einem Drittel bis der Hälfte der Patienten, die am Ende als Nonresponder bezeichnet werden müssen, und sogar 60-80\%, die nicht in die Remission gelangen oder nach Psychotherapie einen Rückfall erleiden (z. B. [40]).

2. Stagnation der Effektivität Trotz intensiver Forschung und der inzwischen als inflationär bezeichneten Entwicklung immer neuer Psychotherapieansätze, welche häufig unter dem Schlagwort „3. Welle“ zusammengefasst werden [53], müssen wir eine Stagnation der Effektivität verzeichnen: Die neuen Therapiemethoden sind den traditionellen meist nicht (wesentlich) überlegen.

3. Kluft zwischen psychotherapeutischer Praxis und Psychotherapieforschung

Diese Kluft ist von Seiten vieler Praktiker gekennzeichnet durch Misstrauen und der Infragestellung der werden, belegen (z. B. [41]). Ihre Effek- 
Relevanz und Übertragbarkeit von Studienbefunden auf ihre Routinebedingungen [57]. Um es methodisch auszudrücken: Die häufig hohe interne Validität von RCTs wirkt sich tatsächlich oft negativ auf die externe Validität (also die Generalisierbarkeit der Ergebnisse auf die Praxis) aus.

\section{Mangelnde Dissemination}

Zudem erreichen die wirksamen Psychotherapien längst nicht alle behandlungsbedürftigen Patienten. So haben $57 \%$ der Personen mit einer validierten Diagnose einer psychischen Störung in Deutschland in den letzten 12 Monaten keine ambulante oder stationäre Hilfe aufgrund ihrer psychischen Beschwerden in Anspruch genommen [36].

5. Wenige Kenntnisse über Wirkmechanismen

Auch wissen wir noch erstaunlich wenig, wie und warum Psychotherapien wirken. In der Psychotherapiedebatte kommen Wampold und Kollegen [60] bzgl. Wirkmechanismen zu dem Schluss, dass vor allem die kontextuellen Faktoren (z. B. Zielkonsensus, Empathie, Allianz) für die Wirksamkeit entscheidend seien und weniger die spezifischen Komponenten (wie z.B. die unterschiedlichen Techniken). Hier herrscht jedoch Forschungsbedarf, da diese Zusammenfassung vor allem auf korrelativen Studien basiert, welche wenig Aufschluss über die zeitliche Abfolge sowie moderierende und mediierende Faktoren geben.

6. Grundsätzliche methodische Probleme

Das Paradigma der evidenzbasierten Medizin wurde auf die Psychotherapieforschung übertragen, sodass auch hier RCTs als Goldstandard der Wirksamkeitsprüfung gelten. Dies ist jedoch problematisch, da beispielsweise in Psychotherapiestudien keine Placebobedingung realisiert werden kann, Therapeuten und Patienten nicht verblindet werden können und für viele konfundierenden Variablen nicht kontrolliert werden kann (vgl. [59]). So stellen die publizierten Effektstärken möglicherweise Überschätzungen dar, wofür auch der
Publikationsbias sowie Powerprobleme verantwortlich gemacht werden können (z.B. [14]).

Diese sechs Punkte weisen eindrucksvoll auf die Notwendigkeit einer Veränderung der Psychotherapieforschung hin: Wir benötigen nicht immer mehr RCTs und Methoden auf dem Markt (wobei es fraglich ist, ob die neuen Methoden wirklich neu sind oder nicht eher altes wiederaufgenommen haben - vgl. „Geschichtsvergessenheit“ [57]). Vielmehr sehen wir die Zukunft darin, die bereits von Gordon L. Paul 1969 aufgeworfene und von Alan E. Kazdin und anderen Forschern immer wieder aufgegriffene Psychotherapieforschungsfrage zu adressieren ([50, S. 44]; vgl. auch [35, 38]):

What treatment, by whom, is most effective for this individual with the specific problem, under which set of circumstances, and how does it come about?

In den letzten Jahrzehnten wurden bereits Konzepte jenseits der störungsspezifischen Psychotherapie entwickelt, welche die von Paul aufgeworfene Frage und somit Konzepte der personalisierten bzw. Präzionstherapie adressieren und im nächsten Absatz zusammenfassend referiert werden. Da bei Ansätzen der personalisierten Medizin der $\mathrm{Pa}$ tient häufig auf molekularbiologische Konstellationen reduziert wird, plädieren wir dafür, in der Psychotherapie dieses Forschungsparadigma auf das konkrete biographische Individuum mit seiner unverwechselbaren Individualität $\mathrm{zu}$ erweitern, welche die biologische Realität natürlich beinhaltet, aber den Einbezug der Person als Ganzes meint (vgl. [30]). Daher präferieren wir (zumindest deutschsprachig) den Begriff „individualisiert“ statt „personalisiert“. Passend in diesem Zusammenhang erscheint auch der Begriff der personenzentrierten Psychotherapie, der zurückgehend auf die Psychotherapie Rogers - den Fokus auf die Erfahrungen und den Lebensraum von Personen, die als Experten für ihr Erleben gesehen werden, setzt (s. auch Leitthema des Kongresses der Deutschen Gesellschaft für Psychiatrie und Psychotherapie, Psychosomatik und Nervenheilkunde 2019).

\section{Neue Ansatzpunkte und Konzepte jenseits der störungsspezifischen Psychotherapie}

Der Erarbeitung und systematischen Prüfung störungsspezifischer Psychotherapien hat insbesondere die Psychiatrie und Psychosomatik die zunehmende Verbreitung psychotherapeutischer Behandlungsansätze zu verdanken. Grundlage hierfür war deren Manualisierung sowie Evidenzbasierung in einer Reihe von RCTs. Allerdings mangelt es trotz Einzug einzelner störungsspezifischer Programme in stationäre Behandlungspläne - z. B. KVT und IPT (interpersonelle Psychotherapie) zur Behandlung der Depression - insgesamt noch an einer breiten Implementierung im Versorgungsalltag. Hierfür dürfte neben der wachsenden Kritik an der traditionellen psychiatrischen Nosologie die unzureichende Berücksichtigung und Anpassung der störungsspezifischen Psychotherapien an Schweregrad, Komplexität und Komorbidität der psychischen Störungen verantwortlich sein. Patienten mit derselben psychischen Diagnose bilden trotz mehr oder weniger hoher phänomenologischer Ähnlichkeit keine homogene Krankheitsgruppe, sondern können hinsichtlich zugrunde liegender Krankheitsmechanismen und ätiologischer Faktoren sehr heterogen sein. Die Identifizierung von im Outcome unterschiedlichen Subtypen (z.B. mit und ohne frühe Traumatisierung [58]) sowie Komorbiditätsraten von bis zu 80 \% (z. B. bei Depression) unterstreichen die Notwendigkeit von mehr Flexibilität und einem höheren Individualisierungsgrad im psychotherapeutischen Vorgehen.

\section{Transdiagnostische Psychotherapien}

Ein Weg in Richtung psychotherapeutische Angebote jenseits nosologischer Krankheitsentitäten sind transdiagnostische Psychotherapien. Beispielsweise zielt das für die emotionalen Störungen formulierte „Unified Protocol for the Transdiagnostic Treatment of Emotional Disorders" auf gemeinsame Merkmale der negativen Affektivität bzw. des Neu- 
Nervenarzt 2019.90:1125-1134 https://doi.org/10.1007/s00115-019-00808-9

(c) Der/die Autor(en) 2019

\section{E.-L. Brakemeier · S. C. Herpertz}

\section{Innovative Psychotherapieforschung: auf dem Weg zu einer evidenz- und prozessbasierten individualisierten und modularen Psychotherapie}

\section{Zusammenfassung}

Psychotherapie ist nachweislich wirksam. Diese Aussage trifft jedoch insbesondere auf den „Durchschnittspatienten" in randomisiertkontrollierten Studien zu. Da trotz der stetigen Entwicklung neuer Therapiemethoden konstant ein beträchtlicher Anteil an Patienten nicht profitiert und die Wirkmechanismen noch zu wenig verstanden werden, gilt es, innovative Psychotherapieforschung zu initiieren, die beide Probleme adressiert. Dabei sollte der aus der somatischen Medizin stammende Gedanke der Personalisierung - oder aus unserer Sicht passender - der Individualisierung bzw. Personenzentrierung aufgegriffen werden. Nach einem Überblick über Weiterentwicklungen der Psychotherapie jenseits störungsspezifischer
Methoden stellen wir als visionäres Ziel psychotherapeutischer Forschung eine evidenz- und prozessbasierte individualisierte und modulare Psychotherapie vor: Jenseits der Syndrome und Störungen sollten möglichst viele biopsychosoziale Charakteristika und die den psychischen Problemen zugrunde liegenden Prozesse bzw. Mechanismen analysiert und in einer individuellen umfassenden funktionellen Analyse gebündelt werden. Auf Basis dieser Funktionsanalyse sollten evidenzbasierte Techniken und Module ausgewählt werden. Das individuelle Ansprechen im Therapieverlauf ist kontinuierlich zu dokumentieren, sodass durch Feedback das weitere therapeutische Vorgehen mitbestimmt wird. Zur Verfolgung dieser Vision werden Studien benötigt, welche an dem individuellen Patienten orientiert sind, die zentralen Wirkmechanismen untersuchen, translational große Datensätze generieren, die auch durch idiographische Analysen ausgewertet werden, sowie die Kluft zwischen Forschung und Praxis mindern und damit zu dem derzeit zu Recht ins Zentrum der Forschung rückenden Paradigma der praxisbezogenen Forschung beitragen.

\section{Schlüsselwörter}

Computationale Psychotherapie · Personalisierte Psychotherapie · Prozessorientierte Therapie · Praxisbezogene Forschung • Biopsychosoziale Charakteristika

\section{Innovative psychotherapy research: towards an evidence-based and process-based individualized and modular psychotherapy}

\section{Abstract}

Psychotherapy has been proven to be effective; however, this statement applies in particular to the "average patient" in randomized controlled trials. As a considerable proportion of patients do not show any benefits despite the constant development of new therapy methods and the mechanisms of action are still too little understood, innovative psychotherapy research has to address both problems. In addition, the idea of personalization that originated in somatic medicine or - from our point of view more appropriately individualization or person-centering should be taken up. After providing an overview of further developments in psychotherapy beyond disorder-specific methods, this article presents an evidence- and process-based individualized and modular psychotherapy as a visionary goal of psychotherapeutic research: Beyond syndromes and disorders, as many biopsychosocial characteristics as possible and the processes and mechanisms underlying the mental problems should be analyzed and bundled in an individual comprehensive functional analysis. Based on this functional analysis, evidence-based techniques and modules should be selected. The individual response during the course of therapy should be continuously documented, so that feedback helps to determine the further therapeutic procedure. In order to pursue this vision, studies are needed that are oriented towards the individual patient, investigate the central mechanisms of action and generate large translational datasets. These should be analyzed by ideographic analyses and reduce the gap between research and practice, thus contributing to the paradigm of a practice research network, which is now consistently moving to the centre of research.

\section{Keywords}

Computational psychotherapy · Personalized psychotherapy - Process-oriented therapy . Practice-related research · Biopsychosocial characteristics rotizismus bei Angststörungen, Zwangsstörungen und Depressionen [2]. Bei all diesen Störungen haben sich Techniken wie Achtsamkeitsübungen, Abbau der Vermeidung emotionaler Erfahrungen und kognitive Neubewertung als wirksam erwiesen [2].

Weitere transdiagnostische Psychotherapiemethoden im Sinne von „common elements treatment approaches“ beziehen sich auf Essstörungen [19] und traumaassoziierte psychische Störungen, bei denen sich Techniken wie Ressourcenaktivierung, kognitive Umstrukturierung und Expositionstechniken als wirksam erwiesen haben [61].

\section{Research Domain Classification}

Mit der Entwicklung der Research Domain Classification (RDoC) psychischer Erkrankungen wurde ein zweiter Weg des psychotherapeutischen Zugangs gebahnt, der jenseits aller nosologischen
Bezüge psychologische und biologische Funktionsstörungen zum Target psychotherapeutischer Interventionen macht. Spezifische RDoC-Ansätze sind beispielsweise solche zur Emotionsregulation [48], die v. a. bei emotionalen Störungen, und solche zu sozial-kognitiven Funktionen, die z. B. bei schizophrenen Psychosen [25] zur Anwendung kommen. So konnte gezeigt werden, dass eine Verbesserung der Emotionsregulation 
$\mathrm{zu}$ einer Verbesserung der depressiven Symptomatik insgesamt führt [21].

Andere zunächst störungsspezifische Ansätze wurden in ihrem Indikationsspektrum schrittweise erweitert und auf die Beeinflussung weiterer Funktionsstörungen ausgerichtet. So hat die dialektisch-behaviorale Therapie (DBT) nicht nur bei der Borderline-Persönlichkeitsstörung, sondern auch bei anderen psychischen Störungen wie bipolaren Störungen und Essstörungen, die sich um eine dysfunktionale Emotionsregulation gruppieren, Wirksamkeit gezeigt. In ähnlicher Weise erweitert sich derzeit der Indikationsbereich der mentalisierungsbasierten Therapie (MBT) auf Patienten mit Psychosen [9] und Depressionen, bei denen sozial-kognitive Störungen im Zusammenhang mit Bindungsproblemen im Zentrum stehen [4].

Die Weiterentwicklung einer funktionsorientierten Psychotherapie könnte sich vorzugweise auf zentrale Funktionsdomänen wie Selbstwert, Identität, Handlungskontrolle und Körperbild (neben der Affekt- und Stressregulation sowie sozialer Kognition) beziehen [32].

\section{Mechanismen- oder prozess- basierte Psychotherapie}

Eher als bei Diagnosen lassen sich bei Funktionsstörungen spezifische Mechanismen auf psychologischer und beginnend auch auf neuronaler Ebene identifizieren. Die sog. mechanismenoder auch prozessbasierte Psychotherapie entspricht Alan E. Kazdins Empfehlungen zur Psychotherapieentwicklung [38], wonach in einem ersten Schritt die Mechanismen bzw. Prozesse zu identifizieren sind, die einer Störung, Subtypen von Störungen oder Funktionsstörungen zugrunde liegen, um hieraus relevante Veränderungsprozesse abzuleiten, die mit geeigneten Methoden messbar sind und einen Zusammenhang zu klinischer Verbesserung zeigen. Bei der Borderline-Persönlichkeitsstörung zielen mechanismenbasierte Interventionen beispielsweise auf die gestörte Emotionsregulation als zentralen Störungsmechanismus und - der Idee einer Neuropsychotherapie [27] folgend auf damit einhergehende präfrontolim- bische Dysfunktionen als grundlegenden Pathomechanismus. Entsprechend konnte bei erfolgreichen Psychotherapien eine Zunahme des Volumens [44] und eine Normalisierung der Funktion im präfrontolimbischen Hirnnetzwerk beobachtet werden, was mit einer Verbesserung der Emotionsregulation sowie der Störung insgesamt einherging [47, 55].

In der internationalen Psychotherapieforschung fordern Hayes und Hofmann den gezielten Einbezug solcher „Kernprozesse“, welche auf der Grundlage testbarer Theorien durch Mediatorenund Moderatorenanalysen identifiziert werden, um eine prozess-orientierte Therapie zu etablieren [28, 35]. Outcomestudien sollten daher nicht nur zu einer Aussage über die Wirksamkeit einer Intervention kommen, sondern vielmehr auch die Moderator- und Mediatorvariablen identifizieren, die vorhersehen helfen, wie bei wem positive Veränderungsprozesse initiiert werden können. Hayes und Hofmann legen in dem ersten Manual zur „process-based CBT" dar, dass insbesondere Techniken aus der KVT und der Akzeptanz- und Commitment-Therapie (ACT) diesen Anspruch erfüllen und gleichzeitig gut miteinander kombinierbar sind [28].

\section{》) Outcomestudien sollten auch Moderator- und Mediatorvariablen identifizieren}

Forschungsdesigns, die konsequent zunächst auf die Identifizierung von Störungsprozessen fokussieren, sind derzeit auf dem Weg, um hieraus Erfolg versprechende Interventionen abzuleiten, deren Wirksamkeit klinisch als auch bezogen auf die Normalisierung dieser Prozesse bzw. Mechanismen (wie z.B. unter Einbezug von Erwartungen [51] oder Biomarkern [32]) getestet werden.

\section{Modulare Psychotherapie}

Die modulare Psychotherapie beschreibt psychotherapeutische Programme, die sich aus eigenständigen funktionellen Einheiten (sog. Modulen) zusammensetzen, die untereinander vielfältig kom- biniert werden können [12]. Ausgangspunkt modularer psychotherapeutischer Programme war die Beobachtung, dass Patienten mit komorbiden Störungen auf störungsspezifische Behandlungsprogramme eine schlechtere Response zeigen [52]. Modulare Psychotherapie entwickelt Kompositionen von Interventionen, die basierend auf der individuellen Fallkonzeption Techniken der allgemeinen Psychotherapie nutzt sowie solche, die validierten störungsspezifischen Programmen entnommen sind. Ziel ist die Veränderung von (sich ggf. selbst aufrechterhaltenden) Pathomechanismen sowie der Aufbau von Veränderungsmechanismen $[6,31]$.

Empirische Unterstützung erfuhr dieser modulare Ansatz v.a. durch die MATCH-Studie (Modular Approach to Therapy for Children), in die Kinder eingeschlossen wurden, die eine Kombination von Angststörungen und Depressionen, aber auch Störungen des Sozialverhaltens zeigten [62].

Während diese Ansätze einen hohen methodischen Komplexitätsgrad von Studiendesigns bei Wirksamkeitsprüfungen implizieren, ist der Implementationserfolg leichter zu erzielen, weil die hohe Flexibilität im Vorgehen die Anpassung an das jeweilige Behandlungssetting erleichtert [17]. Sie eignen sich in besonderer Weise für das psychiatrische Behandlungssetting, das auf wirksame Kurzinterventionen angewiesen ist [32]. Die Auswahl der Module erfolgt flexibel und bedarfsgerecht in Abhängigkeit von den individuellen Problemen des Patienten, seltener den Symptomen und zunehmend den Funktionsstörungen, die dem Leiden zugrunde liegen und auf der Verhaltensebene beobachtbar und messbar sind. Sie werden zu einem individuellen Programm zusammengestellt, das Algorithmen für die Reihenfolge in der Auswahl festlegt.

\section{Ein Beispiel aus der Praxis: "CBASPersonalized"}

Entsprechend dieser Herangehensweise wurde für die Behandlung komplex erkrankter persistierend depressiver Patienten, die Kindesmisshandlung erfahren haben (transdiagnostisches Einschluss- 
kriterium), das individualisierte und modulare stationäre Psychotherapiekonzept CBASPersonalized ${ }^{1}$ konzeptualisiert $[7,54]$. Das Cognitive Behavioral Analysis System of Psychotherapy (CBASP) wurde ursprünglich als störungsspezifische Methode für die Behandlung von Patienten mit persistierenden depressiven Störungen (PDS) entwickelt und integriert Techniken aus verschiedenen Verfahren (• Abb. 1). Bedingt durch sieben RCTs aus unterschiedlichen Arbeitsgruppen $[46,56]$ kann CBASP als „empirically supported treatment" bezeichnet werden.

Da diese Studien jedoch auch zeigen, dass der Anteil an Nonrespondern und Nonremittern relativ hoch ist, der langfristige Verlauf optimierbar erscheint und es einen hohen Prozentanteil an Komorbiditäten unter Patienten mit PDS gibt, wurde CBASP um weitere bereits bewährte, also evidenzbasierte Techniken und Module in Abhängigkeit der individuellen Problematik ergänzt (z.B. Achtsamkeitsübungen und Skills bei Schwierigkeiten der Emotionsregulation, expositionsbasierte Techniken bei komorbiden Angststörungen und Missbrauchserfahrungen). Eine Studie, welche die Wirksamkeit einer solchen, an klassischen CBASP-Techniken ansetzenden "maßgeschneiderten Therapie“ (• Abb. 1) mit der klassischen CBASPTherapie vergleicht, ist in Planung.

\section{Vorschlag für innovative Psychotherapieforschung}

Basierend auf den vorgestellten Konzepten plädieren wir zusammenfassend dafür, die Vision einer evidenz- und prozessbasierten individualisierten und modularen Psychotherapie zu verfolgen. In - Abb. 2 ist unser Vorschlag eines umfassenden und visionären Forschungsprogramms für innovative Psychotherapieforschung zusammengefasst. Diesen Vorschlag werden wir im Folgenden erläutern.

1 auf deutsch individualisiertes CBASP (Unterscheidung der Begriffe "individualisiert" und „personalisiert"s. o.)
Berücksichtigung biopsychosozialer Charakteristika jenseits der Diagnosen

Es werden Targets (also Ansatzpunkte) der Individualisierung bei der Wahl des therapeutischen Vorgehens jenseits der Syndrome und Störungen gesucht, welche im DSM (Diagnostic and Statistical Manual of Mental Disorders) oder ICD (International Statistical Classification of Diseases and Related Health Problems) beschrieben werden. Dabei werden die Patienten als Individuen im Gesamtzusammenhang ihrer biologischen, psychologischen, geistigen, sozialen, ökonomischen, kulturellen und spirituellen Dimensionen betrachtet. Aufgrund der gesellschaftlichen Herausforderungen durch demographischen Wandel und Migration sollten Psychotherapien insbesondere speziell auf die Probleme und Bedürfnisse älterer bzw. hochbetagter Patienten sowie von Patienten mit Migrationshintergrund bzw. Geflüchteten abgestimmt werden.

\section{Computationale Psychotherapie}

Um ein tieferes Verständnis zu erhalten, wie es zu den individuellen psychischen Problemen kam, warum diese aufrechterhalten werden und wie bestimmte Therapien wirken, sollten die biopsychosozialen Prozesse bzw. Mechanismen theoriegeleitet untersucht werden. Bisher bestehen Erklärungsmodelle für psychische Störungen auf unterschiedlichen Ebenen (genetisch, molekular, kognitiv etc.), wobei ein Modell fehlt, welches die verschiedenen Ebenen verbindet und $\mathrm{zu}$ mechanistischen Erklärungen beiträgt. Sowohl zur konzeptionellen Formulierung von Prozessen als auch zur Entwicklung von Methoden zur Detektion individueller Krankheitsprozesse kann die computationale Psychotherapie [45] bzw. Psychiatrie [24] herangezogen werden. Insbesondere sollte zusätzlich $\mathrm{zu}$ nomothetischen Analysemethoden, welche bereits bei der personalisierten Medizin an die Grenzen kommen [23], idiographische Methoden (also die möglichst genaue Analyse von Einzelfällen auf der Basis vieler Daten von dem Individuum) genutzt werden, die so-
Hier steht eine Anzeige. Springer 
Ansatzpunkte

1. Kindesmisshandlung

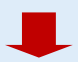

2. Prägungen / Denkschemata

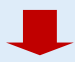

3. Heutige interpersonelle Probleme / Verhaltensdefizite

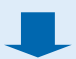

4. Weitere individuelle Probleme
Techniken und Module

$\square$ Liste prägender

Beziehungen

$\neg$ Übertragungshypothese(n)

Kontingente persönliche

Reaktion

$\square$ Interpersonelle

Diskriminationsübung

$\square$ Kiesler Kreis

Situationsanalyse

$\square$ Entsprechende

evidenzbasierte Techniken

und Module

Abb. 1 ॥ Psychopathologische Ansatzpunkte, daran ansetzende klassische CBASP (Cognitive Behavioral Analysis System of Psychotherapy) -Techniken sowie die individualisierte Augmentation mit weiteren Techniken und Modulen gemäß vorliegender Problematik von CBASPersonalized

wohl bei der Individualisierung bereits wertvolle Ergebnisse geliefert haben als auch zu einem besseren Verständnis von Veränderungsmechanismen beitragen [22]. Auch wenn dieser Ansatz für Patienten sehr anspruchsvoll ist, da er langwierige Ecological-Momentary-Assessments(EMA)-Erhebungen beinhaltet und somit die multiple Erfassung von „real-time data“ in der „real world“ sowie auch während der Psychotherapiesitzungen („processes in session“) über eine längere Zeitperiode verlangt, erscheint der Aufwand wegen der gesteigerten Effektivität und des besseren Verständnisses der Wirkmechanismen gerechtfertigt (vgl. [29]).

\section{Funktionsanalyse}

Basierend auf den beiden vorherigen Schritten sollte anschließend ein individuelles, umfassendes Fallkonzept bzw. eine "functional analysis" [34] entwickelt werden. Anhand dieser erfolgt die Auswahl des therapeutischen Vorgehens. Hierfür steht eine ganze Bandbreite an Techniken bzw. Modulen zur Verfügung, welche aus den evidenzbasierten Psychotherapieverfahren und den neueren teilweise bereits evidenzbasierten $\mathrm{Me}$ thoden (wie ACT, DBT, CBASP, Schematherapie, achtsamkeitsbasierte Therapien, mentalisierungsbasierte Therapie, metakognitive Therapie) entnommen werden können [16]. Interessanterweise werden auch zunehmend Psychotherapiemethoden (wieder) eingeführt und beforscht, welche eher einem humanistischen und integrativen ganzheitlichen Menschenbild folgen. Dies betrifft u. a. Ansätze aus der positiven Psychologie wie z.B. die Well-being-Therapie [20]. Schließlich lässt sich eine (Wieder-)Entdeckung der Bedeutung vom Körper in der Psychotherapie beobachten, was sicherlich im engen Zusammenhang mit dem Paradigmenwechsel „embodied turn“ bzw. ,Embodiment“ innerhalb der Psychologie steht.

\section{Psychotherapie als Teil eines Gesamtbehandlungsplans}

Zudem sollte natürlich beachtet werden, dass Psychotherapie meist als Teil eines Gesamtbehandlungsplans untersucht wird (weshalb "psycho" in • Abb. 2 in Klammern gesetzt wurde), was v.a. bedeutet, dass die Kombination mit Psychopharmakotherapie in der Akut- und Erhaltungstherapie zu beachten und weiter zu beforschen ist. Wirksame und innovative Therapiestrategien beziehen zudem die Kombination oder Augmentation von Psychotherapie mit Stimulationsverfahren ein (vgl. $[1,8])$.

\section{Vorhersagemodelle}

Die Auswahl der Techniken und Module sollte evidenzbasiert sein, was durch Metaanalysen zu Patientenmerkmalen als Outcomeprädiktoren bzw. Vorhersagemodelle (bestenfalls generiert durch Big Data) unterstützt werden kann. Ausgehend von den großen Datenpools sollen Algorithmen entwickelt werden, die Profile von Behandlungstechniken aufnehmen, die in erfolgreichen Psychotherapien in definierten Patientenpopulationen zur Anwendung kamen [49]. Um unter Einbezug mehrerer, individueller Faktoren von Patienten die Entscheidungsfindung für die optimale Therapieverfahren evidenzbasiert $\mathrm{zu}$ ermöglichen, wurde der Personalized Advantage Index (z. B. [15]) entwickelt. Dieses Modell, welches eine Vielzahl von Variablen vergleicht und gewichtet, wurde in einigen RCTStudien bereits erfolgreich genutzt, um eine optimale Behandlung für einen Patienten vorherzusagen (Überblick in [13]). Auch bieten komplexe Netzwerkansätze die Möglichkeit, theoriegeleitete Prozesse, welche psychische Probleme auslösen oder aufrechterhalten, beim Individuum zu identifizieren und Vorhersagen über Erfolge verschiedener Behandlungsstrategien zu machen $[29,34]$.

\section{Komplexe Netzwerkanalysen}

Um einen allgemeinen Rahmen zu generieren, schlugen jüngst Hayes, Hofmann und Kollegen vor, die Problemanalyse durch komplexe Netzwerkanalysen mit sozialen/kulturellen evolutionswissenschaftlichen Prinzipien zu verbinden, was sie als „,model of model“ bezeichnen. Prinzipien und Systeme wie Variation, Selektion, Retention sowie Kontext werden mit evolutionären Dimensionen (wie Affekt, Kognition, Aufmerksamkeit, Selbst, Motivation, Verhalten) und Ebenen (physiologisch und sozial/kulturell) verbunden und bieten somit unterschiedlichen therapeutischen Orientierungen eine übergeordnete theoretische Grundlage [29]. 

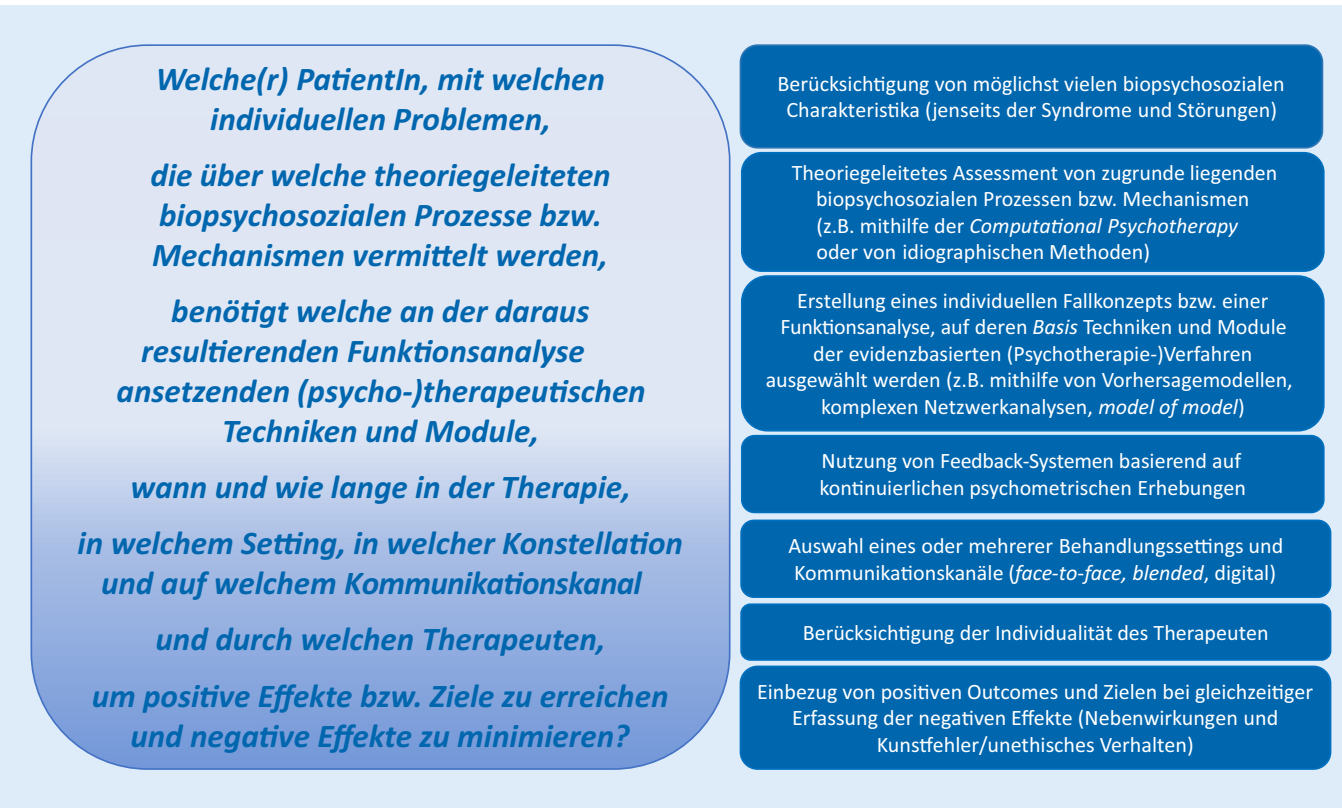

Abb. $2 \triangleleft$ Vorschlag eines Forschungsprogramms zur Erreichung einer evidenzund prozessbasierten individualisierten und modularen Psychotherapie

\section{Feedback}

Die Frage nach dem Zeitpunkt und der Dauer des Einsatzes bestimmter Techniken und Module tangiert die Frage nach der adaptiven Indikation. Der positive Effekt kontinuierlicher psychometrischer Erhebungen - am besten durch EMA unterstützt - und der Rückmeldung (Feedback) der aufbereiteten Informationen zum Therapiefortschritt wurde durch eine Vielzahl von Studien insbesondere für Risikopatienten gezeigt, welche in Gefahr sind, keine oder sogar eine negative Veränderung zu erfahren [40]. Daher sollte Psychotherapieforschung und -praxis zur Qualitätskontrolle und Optimierung anhand systematischer Therapieevaluationen Feedbackprozesse etablieren und implementieren, wie diese beispielsweise in der Arbeitsgruppe um Wolfgang Lutz vorbildlich entwickelt und evaluiert werden (vgl. Trier Treatment Navigator [43]).

\section{Individualität des Therapeuten}

Mehr Beachtung als bisher sollte in der Psychotherapieforschung der Person des Therapeuten geschenkt werden, da Studien darauf hinweisen, dass es beachtliche Therapeuteneffekte gibt (erklären ca. $2,4-8,2 \%$ der Variabilität in den Ergebnissen [37]). Hier wäre Forschung wünschenswert, welche die therapeuti- sche Beziehungsgestaltung experimentell variiert, um herauszufinden, welcher individuelle Therapeut zu welchem individuellen Patienten passt. Zudem sollte zukünftig besserverstanden werden, welche Eigenschaften die sog. "super-shrinks“ im Vergleich zu den "pseudo-shrinks“ haben [11], was auch für die Verbesserung der Aus- und Weiterbildung relevant ist.

\section{Behandlungssetting}

Die Forschungsfragen, in welchem Setting (vollstationär, tagesklinisch, ambulant) und in welcher Konstellation (Einzel, Gruppe, Paar, Familie, System) der individuelle Patient am effektivsten behandelt werden sollte, ist nicht zuletzt bedingt durch den Kosten-Nutzen-Aspekt auch von gesundheitspolitischer Relevanz. Zudem wissen wir mittlerweile, dass viele internet- und mobilbasierte Interventionen (IMIs) wirksam sind [39]. Auch aus einer Kosten-Effektivitäts-Perspektive erscheinen Blended-Interventionen vielversprechend, welche die Stärken der Face-to-face-Psychotherapien mit denen von IMIs kombinieren [18]. Es fehlt jedoch noch ein Verständnis darüber, welche Patienten von den IMIs insbesondere profitieren (und welche eher nicht).

\section{Neue Outcome-Maße}

Schließlich gilt es, auch unsere „primary“ und „secondary“ Outcomes zu erweitern und $\mathrm{zu}$ überdenken: Das einseitige Fokussieren auf die Reduktion der Symptomatologie erscheint als Hauptendpunkt bei diesem Paradigma wenig sinnvoll; vielmehr sollten genau die transdiagnostischen Targets und Prozesse, welche bei der Fallkonzeptualisierung identifiziert wurden, auch als positive Outcomes und Ziele fungieren. Zudem erscheint eine generelle Neuorientierung bei der Feststellung von Therapieeffekten zukunftsweisend. Statt der Messung der Therapieeffekte auf Skalenebene sollten (auch) die Item- und Personenebenen einschließlich der neuen Methoden zu symptomspezifischen Analysen einbezogen werden [5].

Schließlich gilt es, die jahrzehntelang in der Psychotherapie vernachlässigte Nebenwirkungsorientierung aufzugreifen, indem die negativen Effekte (Nebenwirkungen sowie unethisches Verhalten und Kunstfehler) im Prozess erfasst werden, um Psychotherapien nebenwirkungsärmer durchzuführen [33, 42].

\section{Zusammenfassung und Ausblick}

In diesem Beitrag haben wir ein Konzept zukünftiger innovativer Psychothe- 
rapieforschung eingeführt, welches das Ziel einer evidenz- und prozessbasierten individualisierten und modularen Psychotherapie verfolgt. Damit haben wir eine Vision skizziert, welche komplexe, methodisch anspruchsvolle und translationale Forschungsprogramme erfordert. Wissenschaftler (und Praktiker) haben sich jedoch schon "auf den Weg“ gemacht, da etliche bereits zur Beantwortung einiger der in - Abb. 2 aufgeworfenen Forschungsfragen beigetragen haben. Viele dieser bereits publizierten Studien werden jedoch noch durch theoretische und methodische Limitationen eingeschränkt, was zur Folge hat, dass die meisten Ansätze und bereits publizierten Manuale noch nicht gut untersucht, sprich empirisch untermauert oder gar evidenzbasiert sind.

\section{》) Zukünftige Studien müssen sich von Diagnosen als Einschlusskriterien lösen}

Wir sind jedoch der Überzeugung, dass sich dieser Weg jenseits der Verfahrensorientierung und jenseits der „Pferderennen-Studien im Elfenbeinturm“ lohnt. Dafür benötigen wir zukünftig Studien, welche sich von den Diagnosen als Einschlusskriterien lösen und stattdessen an dem individuellen Patienten und seinem Bedarf orientiert sind und den Evidenznachweis hierauf gründen (was übrigens bereits Grawe 1998 [26] gefordert hat). Wir benötigen Studien, welche designt bzw. gepowert sind, um wirklich die zentralen Prozesse bzw. Wirkmechanismen (Moderatoren und Mediatoren) und wirksamen Komponenten untersuchen zu können (statt der absoluten oder relativen Wirksamkeit) und auf diese Weise zur Selektion geeigneter Techniken und Module für den individuellen Behandlungsplan beitragen. Wir benötigen Studien, welche translational große Datensätze (Big Data) generieren, die durch die Anwendung moderner statistischer Verfahren (wie maschinelles Lernen, komplexe Netzwerkanalysen, idiographische Methoden) wertvolle Ergebnisse zur Individualisierung bzw. Personenzentrierung und zu den zugrunde liegenden Prozessen und Wirkmecha- nismen der Psychotherapie liefern. Wir benötigen nicht zuletzt Studien, welche die Kluft zwischen Forschung und Praxis mindern und damit dem derzeit zu Recht ins Zentrum der Forschung rückende Paradigma der praxisbezogenen Forschung (,practice research network" [10]) Rechnung tragen.

Wenn es der Psychotherapieforschung zukünftig gelingt, eine Taxonomie evidenzbasierter Prozesse im Zusammenhang mit evidenzbasierten Techniken und Modulen zu erstellen, kann so statt der unseres Erachtens nicht mehr zeitgemäßen verfahrensorientierten Psychotherapieausbildung eine kompetenzorientierte Psychotherapiequalifikation in der psychotherapeutischen Aus- und Weiterbildung Einzug halten [51]. Wir sehen durch die Novellierung des Psychotherapeutengesetzes mit Neustrukturierung der Aus- und Weiterbildung in Deutschland eine Unterstützung dieses Ansatzes, da im am 26.09.2019 verabschiedeten Gesetz bzgl. des Masterstudiengangs mit Approbation eine "Verfahrensbreite und altersspannenübergreifende psychotherapeutische Qualifikation“ (S. 2) sowie eine Befähigung zur Mitwirkung an der „Weiterentwicklung von psychotherapeutischen Verfahren" (S. 13, §7.1) gefordert wird. Auch tragen Änderungen bei der deutschen Musterweiterbildungsordnung der Fachärzte für Psychiatrie und Psychotherapie dazu bei, dass diese nicht mehr länger nur in Richtlinienverfahren, sondern auch in evidenzbasierten Kurzinterventionen bzw. einzelnen Techniken und Modulen weitergebildet werden. Durch eine kompetente Vermittlung evidenzbasierter Prozesse und Methoden kann eine zukunftsweisende Expertise in der Anwendung einer individualisierten und modularen Psychotherapie erworben werden.

\section{Fazit für die Praxis}

- Psychotherapie ist nachweislich wirksam für den Durchschnittspatienten unserer "Elfenbeinturm-Studien“. Für den individuellen Patienten in der Praxis muss die Psychotherapie (also v. a. die Auswahl der Techniken und Module, jedoch auch des Settings, des Therapeuten etc.) noch besser auf die individuellen Probleme und zugrunde liegenden Prozesse abgestimmt werden.

- Da Psychotherapierende die Wirksamkeit ihrer jeweiligen Psychotherapie "dank" des Positiv-Bias überschätzen und insbesondere negative Verläufe schlecht erkennen, bedarf es evidenzbasierter Heurismen für die Auswahl der jeweiligen Techniken und Module sowie eines Feedbacksystems, welches aus Routinedaten lernend den Therapierenden bei Entscheidungen unterstützt und auf negative Verläufe aufmerksam macht.

- Unsere Vision zukünftiger Psychotherapieforschung zielt deshalb auf eine evidenz- und prozessbasierte individualisierte und modulare Psychotherapie ab.

\section{Korrespondenzadresse}

Prof. Dr. E.-L. Brakemeier

Lehrstuhl Klinische Psychologie und Psychotherapie, Institut für Psychologie, Universität Greifswald

Franz-Mehring-Straße 47, 17489 Greifswald, Deutschland

eva-lotta.brakemeier@uni-greifswald.de

Danksagung. Die Autorinnen danken Herrn Prof. Dr. Stefan Hofmann (Boston University) herzlich für die wertvolle Durchsicht des Manuskriptes.

\section{Einhaltung ethischer Richtlinien}

Interessenkonflikt. E.-L. Brakemeier und S. C. Herpertz geben an, dass kein Interessenkonflikt besteht.

Dieser Beitrag beinhaltet keine Studien an Menschen oder Tieren.

Open Access. Dieser Artikel wird unter der Creative Commons Namensnennung 4.0 International Lizenz (http://creativecommons.org/licenses/by/4.0/deed. de) veröffentlicht, welche die Nutzung, Vervielfältigung, Bearbeitung, Verbreitung und Wiedergabe in jeglichem Medium und Format erlaubt, sofern Sie den/die ursprünglichen Autor(en) und die Quelle ordnungsgemäßnennen, einen Linkzur Creative Commons Lizenz beifügen und angeben, ob Änderungen vorgenommen wurden. 


\section{Literatur}

1. Bajbouj M, Padberg F (2014) A perfect match: noninvasive brain stimulation and psychotherapy. Eur Arch Psychiatry Clin Neurosci 264:S27-33

2. Barlow DH, Farchione TJ, Sauer-Zavala Set al (2017) Unified protocol for transdiagnostic treatment of emotional disorders: therapist guide. University Press, Oxford

3. Barth J, Munder T, Gerger H et al (2013) Comparative efficacy of seven psychotherapeutic interventions for patients with depression: a networkmeta-analysis. PLoS Med 10:e1001454

4. Bateman A, Fonagy P (2015) Borderline personality disorder and mood disorders: mentalizing as a framework for integrated treatment. J Clin Psychol 71:792-804

5. Blanken TF, Van Der Zweerde T, Van Straten A et al (2019) Introducing network intervention analysis to investigate sequential, symptomspecific treatment effects: a demonstration in cooccurring insomnia and depression. Psychother Psychosom 88:52-54

6. Bohus M, Schmahl C, Fydrich T et al (2019) A research programme to evaluate DBT-PTSD, a modular treatment approach for Complex PTSD after childhood abuse. Borderline Personal Disord EmotDysregul 6:7

7. Brakemeier EL, Guhn A, Normann C (in Vorbereitung) Praxisbuch CBASP-Behandlung chronischer Depression und individualisierte Modifikationen für interpersonelle Störungen, 2. Aufl. Beltz, Weinheim

8. Brakemeier EL, Merkl A, Wilbertz G et al (2014) Continuation treatment after electroconvulsive therapy in patients with unipolar depression. Biol Psych 76(3):194-202

9. Brent BK, Holt DJ, Keshavan MS, Seidman LJ, Fonagy P (2014) Mentalization-based treatment for psychosis: linking an attachment-based model to the psychotherapy for impaired mental state understanding in people with psychotic disorders. Isr J Psychiatry Relat Sci 51:17-24

10. Castonguay LG, Barham M, Lutz W, McAleavy A (2013) Practice-oriented research. In: Lambert MJ (Hrsg) Bergin and garfield 's handbook of psychotherapy and behavior change. Wiley, Hoboken, S85-133

11. Castonguay LG, Hill CE (2017) How and why are some therapists better than others: Understanding therapist effects. APA Books, Washington, D.C

12. Chorpita BF, Daleiden EL, Weisz JR (2005) Modularity in the design and application of therapeutic interventions. Appl Prev Psychol 11:141-156

13. Cohen ZD, DeRubeis RJ (2018) Treatment selection in depression. Annu Rev Clin Psychol 14:209-236

14. Cuijpers P, Karyotaki E, Reijnders M, EbertDD (2019) Was Eysenck right after all? A reassessment of the effects of psychotherapy for adult depression. Epidemiol Psychiatr Sci 28:21-30

15. DeRubeis RJ, Cohen ZD, Forand NR et al (2014) The Personalized Advantage Index: translating research on prediction into individualized treatment recommendations. A demonstration. Plos One 9:e83875

16. Dimidjian S, Arch JJ, Schneider RL, Desormeau P, Felder JN, Segal ZV (2016) Considering metaanalysis, meaning, and metaphor: a systematic review and critical examination of "third wave" cognitive and behavioral therapies. Behav Ther 47(6):886-905

17. Durlak JA, DuPre EP (2008) Implementation matters: a review of research on the influence of implementation on program outcomes and the factors affecting implementation. Am J Community Psychol 41:327-350

18. Erbe D, Eichert HC, Riper H, Ebert DD (2017) Blending face-to-face and Internet-based interventions for the treatment of mental disorders in adults: systematic review. J Med Internet Res 19(9):e306

19. Fairburn CG, Cooper Z, Shafran R (2003) Cognitive behaviour therapy for eating disorders: a "transdiagnostic" theory and treatment. Behav Res Ther 41(10):509-528

20. Fava GA (2018) Well-Being Therapie. Eine Kurztherapiezur psychischen Stabilisierung. (Übersetzung durch Brakemeier EL, Schamong I, Bollmann S). Schattauer, Stuttgart

21. Fehlinger T, Stumpenhorst M, Stenzel N, Rief W (2013) Emotion regulation is the essential skill for improving depressive symptoms. J Affect Disord 144:116-122

22. Fisher AJ, Bosley HG, Fernandez KC et al (2019) Open trial of a personalized modular treatment for mood and anxiety. Behav Res Ther 116:69-79

23. Fisher AJ, Medaglia JD, Jeronimus BF (2018) Lack of group-to-individual generalizability is a threat to human subjects research. Proc Natl Acad Sci USA 115:E6106-E6115

24. Friston KJ, Stephan KE, Montague R, Dolan RJ (2014) Computational psychiatry: the brain as a phantastic organ. Lancet Psychiatry 1:148-158

25. Grant N, Lawrence M, Preti A, Wykes T, Cella M (2017) Social cognition interventions for people with schizophrenia: a systematic review focussing on methodological quality and intervention modality. Clin Psychol Rev 56:55-64

26. Grawe K (1998) Psychologische Therapie. Hogrefe, Göttingen

27. Grawe K (2004) Neuropsychotherapie, 2. Aufl. Hogrefe, Göttingen

28. Hayes SC, Hofmann SG (2018) Process-based CBT: the science and core clinical competencies of cognitive behavioral therapy. New Harbinger, Oakland

29. Hayes SC, Hofmann SG, Stanton CE et al (2019) The role of the individual in the coming era of process based therapy. Behav Res Ther 117:40-53

30. Heinze M, Schlimme JE, Kupke C (2013) Personalisierte Psychatrie: Zur Kritik eines Konzepts. Parodos, Berlin

31. Herpertz SC (2017) Modulare Psychotherapie. In: Psychotherapie: Funktions-und Störungsorientiertes Vorgehen. Elsevier, München, S99-104

32. HerpertzSC, CasparF, LiebK(2016) Psychotherapie. Funktions-und störungsorientiertes Vorgehen. Elsevier, München

33. Herzog P, Lauff S, Rief W, Brakemeier EL (2019) Assessing the unwanted - a systematic review of instruments used to assess negative effects of psychotherapy. Brain Behav. e01447. (Epub ahead of print)

34. Hofmann SG, Curtiss J (2018) A complex network approach to clinical science. Eur J Clin Invest 48:e12986

35. Hofmann SG, Hayes SC (2019) The future of intervention science: process-based therapy. Clin Psychol Sci 7:37-50

36. Jacobi F, Höfler M, Strehle J et al (2014) Psychische Störungen in der Allgemeinbevölkerung: Studie zur Gesundheit Erwachsener in Deutschland und ihr Zusatzmodul Psychische Gesundheit (DEGS1MH). Nervenarzt 85:77-87

37. Johns RG, Barkham M, Kellett S, Saxon D (2019) A systematic review of therapist effects: a critical narrative update and refinement to review. Clin Psychol Rev 67:78-93

38. Kazdin AE (2007) Mediators and mechanisms of change in psychotherapy research. Annu Rev Clin Psychol 3:1-27

39. Königbauer J, Letsch J, Ebler P, Ebert DD, Baumeister H (2017) Internet- and mobilebased depression interventions for people with diagnosed depression: a systematic review and meta-analysis. J Affect Disord 223:28-40

40. Lambert MJ (2017) Maximizing psychotherapy outcome beyond evidence-based medicine. Psychother Psychosom 86:80-89

41. Lambert MJ (2013) The efficacy and effectiveness of psychotherapy. In: Lambert MJ (Hrsg) Bergin and Garfield's handbook of psychotherapy and behavior change. Wiley, New York

42. Linden M, Strauß B, Brakemeier EL et al (2018) Definition und Entscheidungsschritte in der Bestimmung und Erfassung von Nebenwirkungen von Psychotherapie. Psychother Psychosom Med Psychol 68:377-382

43. Lutz W, Rubel JA, Schwartz B, Schilling V, Deisenhofer AK (2019) Towards integrating personalized feedback research into clinical practice: Development of the Trier Treatment Navigator (TTN). Behav Res Ther 120:103438

44. Mancke F, Schmitt R, Winter D et al (2018) Assessing the marks of change: how psychotherapy alters the brain structure in women with borderline personality disorder. J Psychiatry Neurosci 43:171-118

45. Moutoussis M, Shahar N, Hauser TU, Dolan RJ (2018) Computation in psychotherapy, or how computational psychiatry can aid learningbased psychological therapies. Comput Psychiatr 2:50-73

46. Negt P, Brakemeier EL, Michalak M et al (2016) The treatment of chronic depression with cognitive behavioral analysis system of psychotherapy:a systematic review and meta-analysis of randomizedcontrolled clinical trials. Brain Behav 6:e486

47. Niedtfeld I, Kirsch P, Schulze L, Herpertz SC, Bohus M, SchmahlC(2012) Functional connectivity of pain mediated affect regulation in borderline personality disorder. Plos One 7(3):e33293

48. o'Toole MS, Mennin DS, Fresco DM (2014) Emotion regulation therapy: an experiential approach to chronic anyiety and recurring depression. In: Thoma NC, McKay D (Hrsg) Working with emotion in cognitive-behavioral therapy. Techniques for clinical practice. Guilford, New York, S 310-348

49. Owen J, Imel ZE (2016) Introduction to the special section "Big'er' Data": Scaling up psychotherapy research in counseling psychology.JCouns Psychol 63:247-248

50. Paul GL (1969) Behavior modification research: design and tactics. In: Franks CM (Hrsg) Behavior therapy: appraisal and status. McGraw-Hill, New York, S29-62

51. Rief W (2019) Zur Zukunft der Psychotherapie in Deutschland und die Frage nach der Verfahrensorientierung und-integration. PTJ 3:261-268

52. Riper H, Andersson G, Hunter SB et al (2014) Treatment of comorbid alcohol use disorders and depression with cognitive-behavioural therapy and motivational interviewing: a meta-analysis Addiction 109:394-406

53. Rubel JA, Lutz W (2016) „Personalisierung statt Verfahrensinflation" - Oder: Warum die Psychotherapieforschung in Deutschland ausgebaut werden sollte/könnte/müsste? Teil 2. Psychother Psychosom Med Psychol 66:463-464 
54. Schamong I, Bollmann S, Brakemeier EL (2017) Behandlung der chronischen depression - das Cognitive Behavioral Analysis System of Psychotherapy (CBASP). Ärztl Psychother 12:142-151

55. Schmitt R, Winter D, Niedtfeld I, Herpertz SC, Schmahl C (2016) Effects of psychotherapy on neuronal correlates of reappraisal in female patients with borderline personality disorder. Biol Psychiatry Cogn Neurosci Neuroimaging 1:548-557

56. Schramm E, Kriston L, Zobel I et al (2017) Effect of disorder-specific vs nonspecific psychotherapy for chronic depression: a randomized clinical trial. JAMA Psychiatry 74:233-242

57. Strauss B (2019) Innovative Psychotherapieforschung - Wo stehen wir und wo wollen wir hin? Psychother J 18:4-10

58. Targum SD, Nemeroff CB (2019) The effect of early life stress on adult psychiatric disorders. Innov Clin Neurosci 16:35-37

59. Tschuschke V, Freyberger HJ (2015) Zur aktuellen Situation der Psychotherapiewissenschaft und ihrer Auswirkungen - eine kritische Analyse der Lage. Z Psychosom Med Psychother 61:122-138

60. Wampold BE, Imel ZE, Flückiger C (2018) Die Psychotherapie-Debatte: Was Psychotherapie wirksam macht. Hogrefe, Bern

61. Weiss WM, Murray LK, Zangana GA, Mahmooth Z, Kaysen D, Dorsey S, Lindgren K, Gross A, Murray SM, Bass JK, Bolton P (2015) Community-based mental health treatments for survivors of torture and militant attacks in Southern Iraq: a randomized control trial. BMC Psychiatry 15:249

62. Weisz JR, Chorpita BF, Palinkas LA et al (2012) Testing standard and modular designs for psychotherapy treating depression, anxiety, and conduct problems in youth: a randomized effectiveness trial. Arch Gen Psychiatry 69:274-282

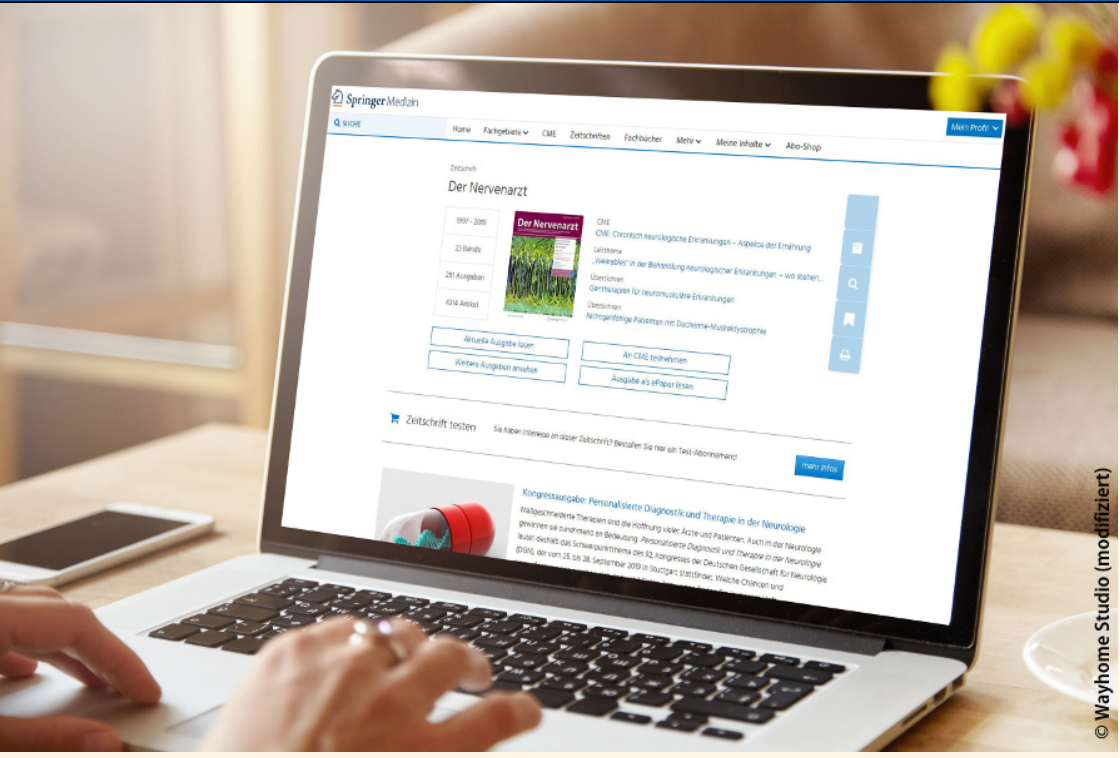

Auch online Zugang zu allen Beiträgen von Der Nervenarzt

Wussten Sie, dass Sie als Abonnent dieser Zeitschrift automatisch online auf das gesamte Beitragsarchiv zugreifen können?

Als Abonnent von Der Nervenarzt erhalten Sie zwölf mal im Jahr Ihre Ausgabe nach Hause geliefert.

Damit nicht genug: Im Abonnement ist auBerdem der Zugriff auf das gesamte OnlineArchiv lhrer Zeitschrift inbegriffen.

\section{Und so einfach geht es:}

Registrieren Sie sich einmal über www.springermedizin.de/register:

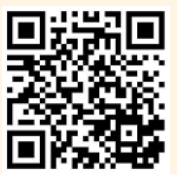

Über diesen QR-Code schnell

und einfach registrieren

Bei der Registrierung geben Sie einfach Ihren Vor- und Nachname und Lieferadresse wie beim Abonnement der Zeitschrift (siehe Adressaufkleber auf Ihrem Heft) an. So kann im System die Zugehörigkeit zu Ihrer Zeitschrift sichergestellt werden.
Aufgrund des Heilmittelwerbegesetzes dürfen die Inhalte der Website nur medizinischen Fachkreisen zur Verfügung gestellt werden. Bei der Anmeldung bitten wir Sie deshalb einen Berufsnachweis vorzulegen. Bei Medizinern mit Mitgliedschaft in der deutschen Ärztekammer reicht die einheitliche Fortbildungsnummer (EFN). Alternativ schicken Sie eine Bestätigung des Arbeitgebers, Studiennachweis oder andere Zeugnisse ganz unkompliziert an kundenservice@springermedizin.de.

Mit Benutzername und Passwort haben Sie außerdem Zugang zu den freien Inhalten auf den Seiten von: https://www.springermedizin.de/ https://www.aerztezeitung.de/

Sollten Fragen oder Probleme auftauchen, wenden Sie sich einfach an Ihren Kundenservice:

kundenservice@springermedizin.de 